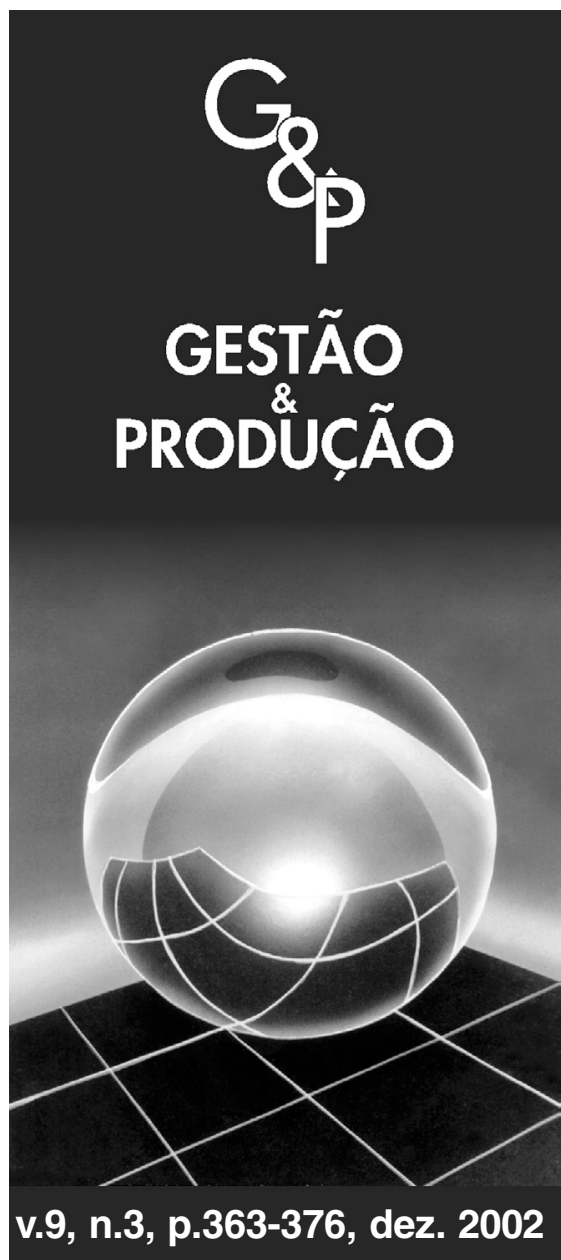

\title{
INTELIGÊNCIA ARTIFICIAL: UMA APLICAÇÃO EM UMA INDÚSTRIA DE PROCESSO CONTÍNUO
}

\author{
Miguel Afonso Sellitto \\ Centro de Ciências Exatas e Tecnológicas, \\ Universidade do Vale do Rio dos Sinos, Unisinos, \\ Av. Unisinos, $s / n$, \\ CEP 93022-000, São Leopoldo, RS, \\ e-mail: miguelsv@terra.com.br
}

Resumo

Este trabalho descreve uma aplicação da lógica fuzzy de controle e do CBR (Raciocínio Baseado em Casos) na indústria de processo contínuo. Essas técnicas são discutidas dentro do campo de conhecimentos da Inteligência Artificial, associadas ao processo de tomada de decisões empresariais. A Inteligência Artificial é apontada como um campo de conhecimentos que pode apoiar a tomada de decisões de um modo mais simples e mais preciso do que outros métodos, tais como a modelagem e a gestão por indicadores. As etapas para a construção de um sistema especialista, construído principalmente a partir de experiências empíricas humanas, também são discutidas. $O$ trabalho se encerra apresentando uma rotina de tomada de decisão em um processo termoquímico na indústria cimenteira conduzida por um sistema especialista baseado em CBR e lógica fuzzy, e uma discussão sobre resultados comparados com operadores humanos nas mesmas condições.

Palavras-chave: lógica fuzzy na indústria de processo contínuo, Inteligência Artificial na engenharia de produção, sistemas especialistas na engenharia de produção, CBR na engenharia de produção. 


\section{Introdução}

No cenário competitivo atual, construir e gerenciar conhecimento de apoio a especialistas no controle de processos industriais pode ser útil para uma organização de fabricação, principalmente para processos que ocorram sob incertezas e com dados incompletos.

$\mathrm{O}$ controle manual em um processo industrial contínuo é exercido instante a instante, por intermédio de decisões que exigem do especialista o conhecimento necessário para relacionar ações e resultados. Exigem ainda o acompanhamento da operação, algumas pilotagens nas variações operacionais, experiência e segurança suficientes para assumir riscos em situações extremas.

A Inteligência Artificial é um campo de conhecimentos que oferece modelos de apoio à decisão e ao controle com base em fatos reais e conhecimentos empíricos e teóricos, mesmo que apoiados em dados incompletos. O objetivo deste trabalho é apresentar a aplicação de alguns conceitos do campo da Inteligência Artificial no desenvolvimento e implantação, a partir do conhecimento de especialistas, do controle de um processo do tipo contínuo, relevante na indústria de cimento: a clinquerização.

\section{Revisão bibliográfica: a Inteligência Artificial}

Inteligência é a demonstração por resultados de princípios coerentes, em escala de tempo verificável: a natureza é inteligente em escala de tempo inacessível para os humanos. O oposto da inteligência é o caos: em um sistema caótico, duas entradas muito próximas resultam em duas saídas sem qualquer conexão (Conai, 1994).

Para McCarthy (2002), inteligência é a parte computacional da habilidade de alcançar objetivos, percebendo-se diversos tipos e graus de inteligência em pessoas, em muitos animais e em máquinas.
Ao menos cinco escolas filosóficas se propõem a descrever a inteligência (Conai, 1994):

- Estruturalista: há mecanismos que realizam as tarefas, basta descobri-los.

- Conexionista: há processos simples que se auto-organizam, basta descobri-los.

- Genética: há a inteligência e o caos, basta separá-los por seleção natural, como faz a natureza, porém em escala de tempo adequada.

- Fenomenológica: tudo é caótico, em avanço paralelo. Ao se dar uma sintonia momentânea, dá-se a comunicação. Não se conhece chave para repetir a inteligência.

- Metafísica: só é possível compreender o intelecto. A inteligência pertence a outra dimensão, inacessível por meios intelectuais.

Para Arariboia (1988), a Inteligência Artificial é um campo que usa técnicas de programação que procuram, por intermédio de máquinas, resolver problemas do mesmo modo que um ser humano os resolveria. Para Nikolopoulos (1997), a Inteligência Artificial é um campo de estudos multidisciplinar, originado da computação, da engenharia, da psicologia, da matemática e da cibernética, cujo principal objetivo é construir sistemas que apresentem comportamento inteligente e desempenhem tarefas com um grau de competência equivalente ou superior ao grau com que um especialista humano as desempenharia. Para McCarthy (2002), a Inteligência Artificial é a ciência e a tecnologia de construção de máquinas inteligentes, especialmente programas de computador. Relaciona-se com o uso de computadores para o entendimento e a exploração da inteligência humana, não se limitando, porém, a métodos biologicamente observáveis. Para Arariboia (1988), as técnicas de Inteligência Artificial procuram imitar mecanismos da natureza por intermédio de mecanismos tecnológicos cujo desenvolvimento foi baseado em mecanismos naturais. 
Diversas técnicas e aplicações estão disponíveis no campo da Inteligência Artificial. Uma referência que remete a outras fontes é encontrada em McCarthy (2002). Para o objetivo deste trabalho são suficientes os conceitos de sistemas especialistas, $C B R$ e lógica fuzzy.

\subsection{Sistemas especialistas, $C B R$ e lógica fuzzy}

Sistemas especialistas são sistemas computacionais que resolvem problemas de forma similar ao modo como um especialista humano os resolveria, possuindo capacidade de decisão em campos específicos do conhecimento. Um sistema especialista resolve problemas em uma área limitada de conhecimento, não se devendo esperar do mesmo que possa ter capacidade de resolver qualquer tipo de problema.

Os sistemas especialistas buscam a resposta e aprendem com a experiência, resolvendo problemas por análise inferencial, a partir de sintomas e intensidades aleatórios e apoiados em bases de conhecimento que podem, inclusive, ser transferidas. Os sistemas especialistas possuem: (i) um banco de conhecimentos que contém fatos, regras e padrões; (ii) um dispositivo de inferência capaz de tomar decisões; (iii) uma linguagem na qual as regras são escritas; (iv) um organizador que inclui o dispositivo de inferência, o gerenciador da base de conhecimento e as interfaces de usuários (Nikolopoulos, 1997; Rabuske, 1995).

O Raciocínio Baseado em Casos $(C B R)$ é uma metodologia que resolve novos problemas adaptando soluções conhecidas de problemas antigos, ou seja, usando conhecimento gerado em experiências passadas. Um novo problema é resolvido encontrando um caso similar já resolvido no passado e reutilizando a solução, devidamente adaptada.

O CBR é uma abordagem incremental para a aprendizagem, capaz de acumular conhecimento a partir de casos bem-sucedidos e recuperáveis de uma base de casos, na qual novos casos são gerados e casos semelhantes são combinados. $\mathrm{O}$ ciclo $C B R$ de aprendizado pode ser representado pelos 4 RE da Figura 1: (i) recuperar casos similares ao atual; (ii) reutilizar uma ou mais das soluções; (iii) revisar a solução proposta para o caso atual; (iv) registrar a nova solução como mais um caso na base de casos (Aamodt \& Plaza, 1994; Corchado et al., 2001).

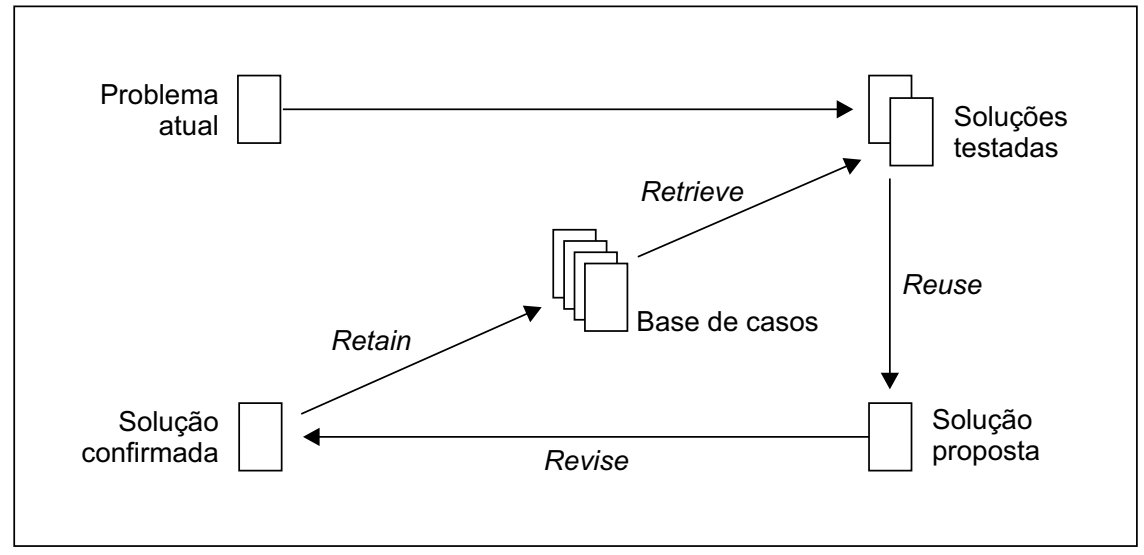

Figura 1 - O ciclo CBR de aprendizado. (Fonte: Corchado et al., 2001.) 
Um problema novo pode ser relacionado a casos armazenados por intermédio de uma função de similaridade. Uma função de similaridade gera uma saída entre 0 e 1 que indica o quanto dois casos são similares, a partir de premissas e de ponderações pertinentes ao objetivo da análise. Uma função de similaridade pode ser do tipo:

$$
S(T, S)=\sum_{i=1}^{n} f\left(T_{i}, S_{i}\right) \cdot w_{i}, \text { em que: }
$$

- T é o caso-alvo;

- S é o caso-fonte, residente na base de casos;

- N é o número de atributos considerados na indexação;

- f é uma função de similaridade a definir;

- w é a importância relativa do atributo $\left(\Sigma \mathrm{w}_{\mathrm{i}}=1\right)$.

O caso-alvo e o caso-fonte devem ser descritos por dimensões originadas de variáveis quantitativas normalizadas ou variáveis categóricas qualificadas. Cada dimensão deve assumir os valores 0 e 1 para os extremos do intervalo de validade, tornando os casos comparáveis. $\mathrm{O}$ módulo da diferença entre dimensões informa o quanto cada dimensão afasta o caso-alvo do casofonte. Se as dimensões tiverem importância diferente, obtêm-se coeficientes de ponderação, por exemplo, por opiniões de especialistas. A função de similaridade toma o complemento de 1 do somatório dos módulos das diferenças, ponderados pelas importâncias relativas das dimensões. Casos iguais terão similaridade igual a 1; casos opostos terão similaridade nula. Os casos com maior similaridade são os candidatos a formar a nova solução e gerar um novo caso-fonte.

A lógica fuzzy (difusa) é uma técnica que pode resolver problemas de modelagem complexa, com aspectos quali e quantitativos, sujeitos a variações probabilísticas relevantes ou descritos por bases de dados diferentes e incompletas. Seu processo decisório se baseia em variáveis lingüísticas que simulam e replicam elementos do pensamento humano, principalmente em bases comparativas, tais como mais alto, mais frio, melhor; ou vagas, tais como alto, baixo, bom (Kacprzyk, 1997).

Antes da lógica fuzzy, exemplifica-se a lógica crisp (abrupta) (Oliveira Jr., 1999).

Sejam pessoas com 1,75 m, 1,85 m e 1,90 m de altura e seja superar $1,80 \mathrm{~m}$ de altura o critério para pertencer ao conjunto dos altos. Pela lógica crisp, o indivíduo com 1,75 m não pertence ao conjunto dos altos e pertence ao conjunto dos não-altos. Os indivíduos com 1,85 $\mathrm{m}$ e $1,95 \mathrm{~m}$ pertencem ao conjunto dos altos $\mathrm{e}$ não pertencem ao conjunto dos não-altos. $\mathrm{O}$ modo pelo qual um indivíduo passa a pertencer ao conjunto é abrupto, ou seja, ao superar o limiar de pertinência o indivíduo é totalmente admitido no conjunto.

A lógica fuzzy assinala a diferentes indivíduos diferentes graus de pertinência a conjuntos adjacentes, de acordo com o grau de superação da condição de pertinência. A transição para a pertinência ao conjunto é gradual, podendo um indivíduo intermediário pertencer aos dois conjuntos, de modo difuso. O grau de pertinência a um conjunto é tanto maior quanto mais afastado está esse indivíduo da fronteira. No exemplo, os indivíduos de $1,85 \mathrm{~m}$ e $1,95 \mathrm{~m}$ pertencem ao conjunto dos altos, porém o primeiro pertence mais ao conjunto dos nãoaltos do que o segundo e o segundo pertence mais ao conjunto dos altos do que o primeiro.

Define-se então a função de pertinência (membership function) a conjuntos adjacentes.

Para o exemplo, seja a função de pertinência da Figura 2. Um indivíduo de $1,80 \mathrm{~m}$ de altura tem um grau de pertinência 0,5 ao conjunto dos altos e um grau de pertinência 0,5 ao conjunto dos não-altos. Um indivíduo com 1,65 m tem grau de pertinência 0 ao conjunto dos altos e de 1 ao conjunto dos não-altos. Finalmente, um indivíduo com $1,95 \mathrm{~m}$ tem grau de pertinência 1 ao conjunto dos altos e de 0 ao conjunto dos não-altos.

Ainda para o exemplo, seja a função de pertinência: $\mathrm{F}$ (alto) $=\mathrm{A} ; \mathrm{F}$ (não-alto) $=\mathrm{B}$.

Avaliando-se a função pela lógica crisp, temse $\mathrm{F}(1,725)=\mathrm{B}$ e $\mathrm{F}(1,875)=\mathrm{A}$. Avaliando-se a função pela lógica fuzzy, tem-se $\mathrm{F}(1,725)=$ $0,75 \mathrm{~B}+0,25 \mathrm{~A}$ e $\mathrm{F}(1,875)=0,25 \mathrm{~B}+0,75 \mathrm{~A}$. 


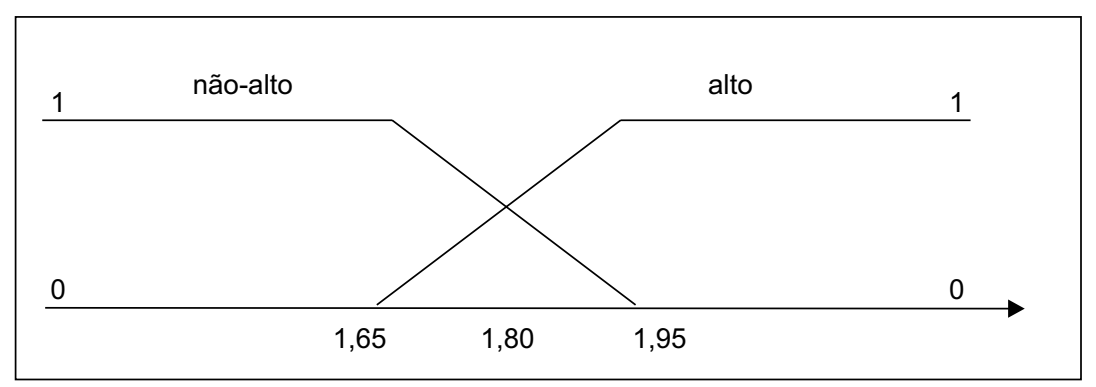

Figura 2 - Função de pertinência difusa para o exemplo.

\subsection{Controladores de processos industriais baseados em lógica fuzzy}

O controle de processos industriais pela lógica fuzzy foi proposto por Mamdani e seus colaboradores, usando a abordagem de Zadeh para conjuntos difusos e incluindo variáveis linguiísticas, sentenças condicionais difusas e regras de inferências composicionais.

Os requisitos que tornam indicado o uso da lógica fuzzy para o controle de um processo industrial são (Kacprzyk, 1997):
- Não existe um modelo do processo a controlar, por ser demasiado complexo ou demasiado custoso seu desenvolvimento.

- O processo é bem controlado por especialistas humanos.

- Os especialistas humanos conseguem verbalizar as regras que usam nas ações manuais de controle do processo.

Um processo industrial em tais condições pode ser controlado por um sistema especialista baseado em lógica fuzzy, como o sistema representado na Figura 3.

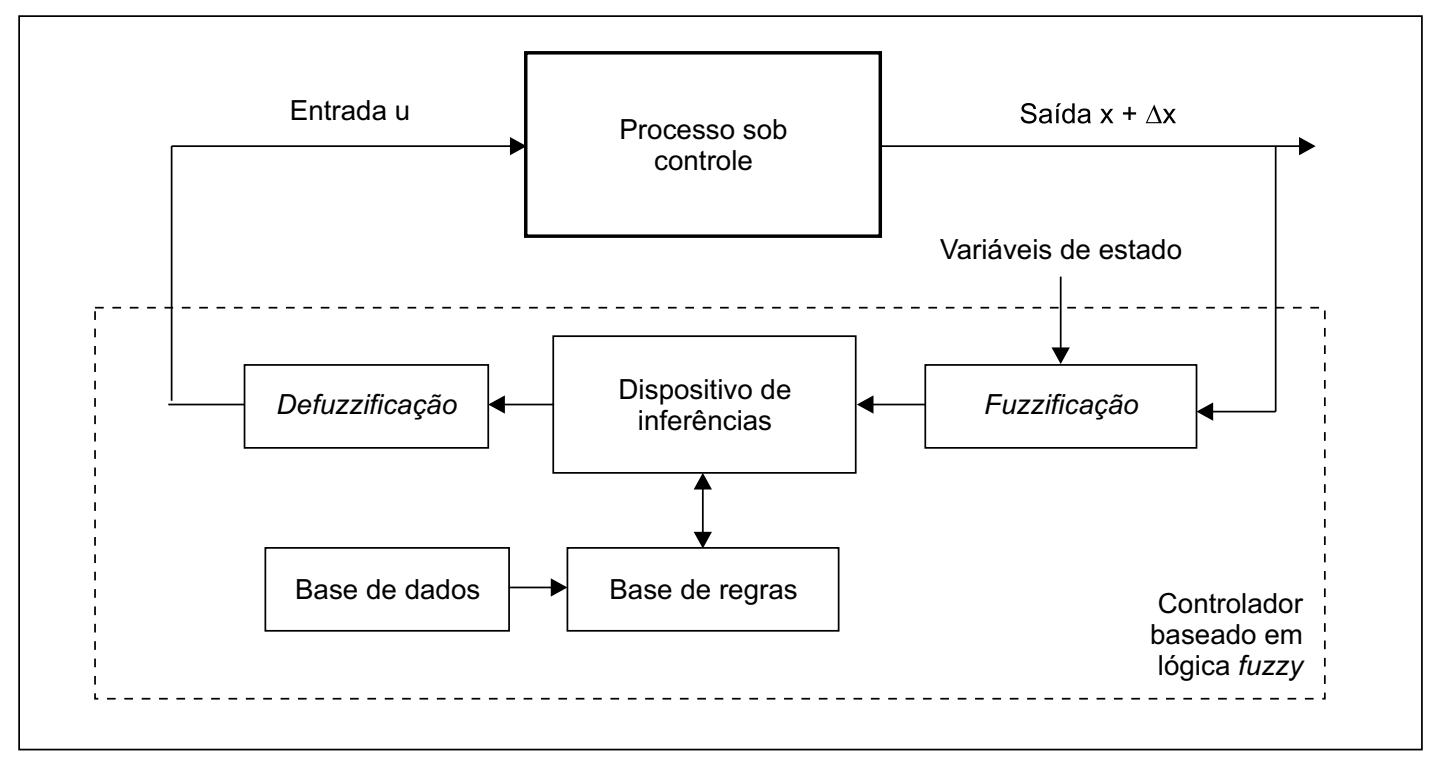

Figura 3 - O uso da lógica fuzzy no controle de processos industriais [adaptado de Kacprzyk (1997)]. 
A base de conhecimento consiste em uma base de regras e em uma base de dados que incorpora o conhecimento do processo, geralmente representado por uma estrutura linguiística quase natural, com termos como: alto, médio, baixo, sobe um pouco, desce muito, etc. Na base de conhecimento se define um universo de discurso consistindo nos termos a serem usados, em escala graduatória, composta por um número ímpar de estágios, o que facilita a compreensão humana. Por exemplo, para cinco estágios, um universo de discurso apropriado para os termos linguísticos seria como na Figura 4. Nessa figura também se têm as correspondentes funções de pertinência fuzzy, associadas a cada um dos valores lingüísticos e normalizadas em um universo de discurso $[-100 \%, 100 \%]$ da variável. O número de valores lingüísticos, o espaço, a sobreposição e o formato das funções de pertinência são definidos durante o projeto do sistema e dependem do grau de conhecimento que se tem do problema (Kacprzyk, 1997).

O processo é controlado por uma variável controlada e seu gradiente $(x+\Delta x)$, manipulada por uma variável de controle $\Delta \mathrm{u}$. A base de regras é construída a partir de depoimentos dos especialistas, que articulam verbalmente as relações entre as variações na variável de controle e as variações esperadas na variável controlada. Essas articulações são representadas na base de regras de modo compreensível pelos especialistas e utilizável pelo controlador fuzzy.
Um modo de representação é o uso de sentenças condicionais (Kacprzyk, 1997):

- IF $\mathrm{x}=\mathrm{NS}$ AND $\Delta \mathrm{x}=\mathrm{NS}$ THEN $\Delta \mathrm{u}=\mathrm{PB}$; ou

- IF $\mathrm{x}=\mathrm{NS}$ AND $\Delta \mathrm{x}=$ NS THEN $\Delta \mathrm{u}=$ $\mathrm{h}_{1,1}(\mathrm{x}, \Delta \mathrm{x})$.

Outro modo é o uso de uma matriz cujas entradas são as grandezas x e $\Delta x$ e a saída é a grandeza $\Delta \mathrm{u}$, contendo todas as possibilidades de cruzamento entre as variáveis. Como um controle real se vale de $\mathrm{n}$ variáveis de controle $\mathrm{e}$ $m$ variáveis controladas, as relações entre as variáveis são representadas em tabelas múltiplas. Este é o formato adotado no caso em estudo.

A base de dados inclui o formato das funções de pertinência, os domínios das variáveis e fatores de escala, empregados na defuzzificação. Caso o sistema preveja o aprendizado com o próprio processo, ou seja, modificam-se as regras a partir do resultado de sua aplicação, a base de dados deverá reter também os parâmetros de desempenho do processo.

Para o processo de fuzzificação, o controlador mede os valores e os gradientes das variáveis controladas e de certas variáveis auxiliares, as variáveis de estado, que antecipam o desempenho do controle. De acordo com as funções de pertinência das variáveis e dos gradientes, o controlador calcula um valor fuzzy, que será usado na inferência da variação da variável de controle.

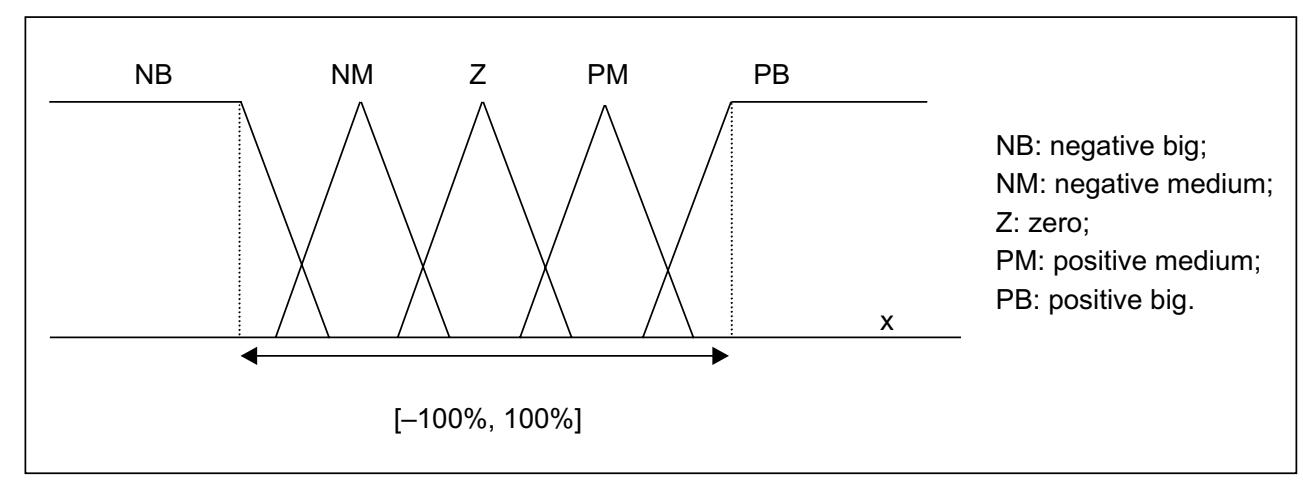

Figura 4 - Variáveis lingüísticas [adaptado de Kacprzyk (1997)]. 
O valor inferido é imposto ao processo em unidades de engenharia, convertidas pelos fatores de escala residentes na base de dados. O valor imposto é a variação do setpoint do processo, inferido, somado ao valor atual, o setpoint do controlador.

Para Kacprzyk (1997), a força da lógica fuzzy como lógica de controle de processos industriais reside em sua aplicação a casos em que não se tem um modelo, mas o processo é operado satisfatoriamente por especialistas. Pela prática da engenharia de produção afirma-se que este é o caso de muitos processos relevantes na indústria de processo contínuo.

\section{Uma aplicação na indústria de processo contínuo}

A seguir descreve-se uma aplicação da lógica fuzzy de controle na indústria de processo contínuo. A aplicação ocorreu em um processo de fabricação de clínquer, matéria-prima usada na produção de cimento. A clinquerização é um processo termoquímico operado manualmente de modo satisfatório e de difícil modelagem exata, o que satisfaz os requisitos de Mamdani.

Para que se contextualize a clinquerização, descreve-se o processo de fabricação de cimento.

\subsection{A clinquerização e o processo de fabricação de cimento}

A seguir descrevem-se as etapas e na Figura 3 apresenta-se um fluxograma de um processo típico de fabricação de cimento (Farenzena, 1995).

Mineração: é o processo de desmonte, extração e remoção dos minerais que constituem a matéria-prima do cimento, normalmente calcário, argila e material estéril. Reduz-se a granulometria por intermédio da fragmentação e cisalhamento em britagens e forma-se a pilha de pré-homogeneização, cuja função é préadequar os parâmetros químicos do material, por intermédio da dosagem de calcários ou argilas extraídos de diversas fontes.
Moagem de cru: O material extraído da pilha é seco e moído em moinhos tubulares ou de rolos, transformando-se em uma mistura fina, a farinha. Na moagem de cru se ajustam os parâmetros físico-químicos da farinha por intermédio da alimentação e dosagem de diversos tipos de calcário. A farinha produzida é armazenada em silos, onde passa por novo processo de homogeneização. A homogeneidade química da farinha é importante para a clinquerização, na qual ocorre a assimilação e a combinação da farinha com o combustível.

Clinquerização: É um processo de aquecimento e tratamento térmico controlado, a até $1.450^{\circ} \mathrm{C}$, que provoca reações termoquímicas na farinha, originando o clínquer. Os materiais se deslocam em oposição aos gases da combustão, que transferem calor por contato às torres de ciclones. A tiragem é realizada por exaustores com tomada de ar a jusante do forno e recuperação da farinha residual em precipitadores eletrostáticos. A fonte de calor é o queimador, a montante do forno, alimentado por carvão moído complementado por casca de arroz, óleos pesados, gás natural ou coque petroquímico. Um parâmetro relevante no controle do forno é o $\mathrm{NO}_{\mathrm{x}}\left(\mathrm{NO}+\mathrm{NO}_{2}\right)$, principalmente por seu impacto ambiental.

Moagem de cimento: A moagem do cimento se dá em circuito fechado, cujo equipamento principal é o moinho tubular de bolas. As matérias-primas são alimentadas por balanças dosadoras de vazão (ton/h), conforme os teores da matéria-prima disponível e o tipo de produto. $\mathrm{O}$ arraste de material ao longo das câmaras é feito por um exaustor, cuja tiragem arrasta o material moído por corpos moedores, que quebram, trituram e misturam os componentes, formando uma massa pulvurulenta e homogênea. Ao atingir a granulometria de saída, é elevado por um transportador vertical e classificado. A fração fina se dirige aos silos de produto pronto, enquanto a fração grossa volta para ser moída até a granulometria especificada. A medição da fração de retorno é importante no ajuste do processo. 
Ensacagem: O cimento produzido na fase de moagem é conduzido por intermédio de transporte mecânico ou pneumático até um conjunto de silos, onde fica protegido da umidade ambiental. A técnica de ensacagem depende do tipo de veículo que será empregado para retirar o produto da fábrica. As embalagens disponíveis são de 50 e $25 \mathrm{~kg}$ ou a granel.

\subsection{Descrição da aplicação}

O processo de clinquerização é representado na Figura 6. As variáveis de controle são a alimentação de matéria-prima, a exaustão e o combustível, doravante chamados de feed, fan e fuel, e as variáveis de estado são a temperatura da zona de queima, a BZT (Burning-Zone Temperature), o teor de oxigênio na queima, o $\mathrm{O}_{2}$ e a temperatura do fundo do forno, a BET(Back-End Temperature). As variáveis controladas são a produtividade em toneladas por hora e/ou a eficiência energética em kilocalorias por grama de produto acabado.

A lógica de controle do processo de clinquerização reside em um controlador eletrônico baseado em lógica fuzzy.

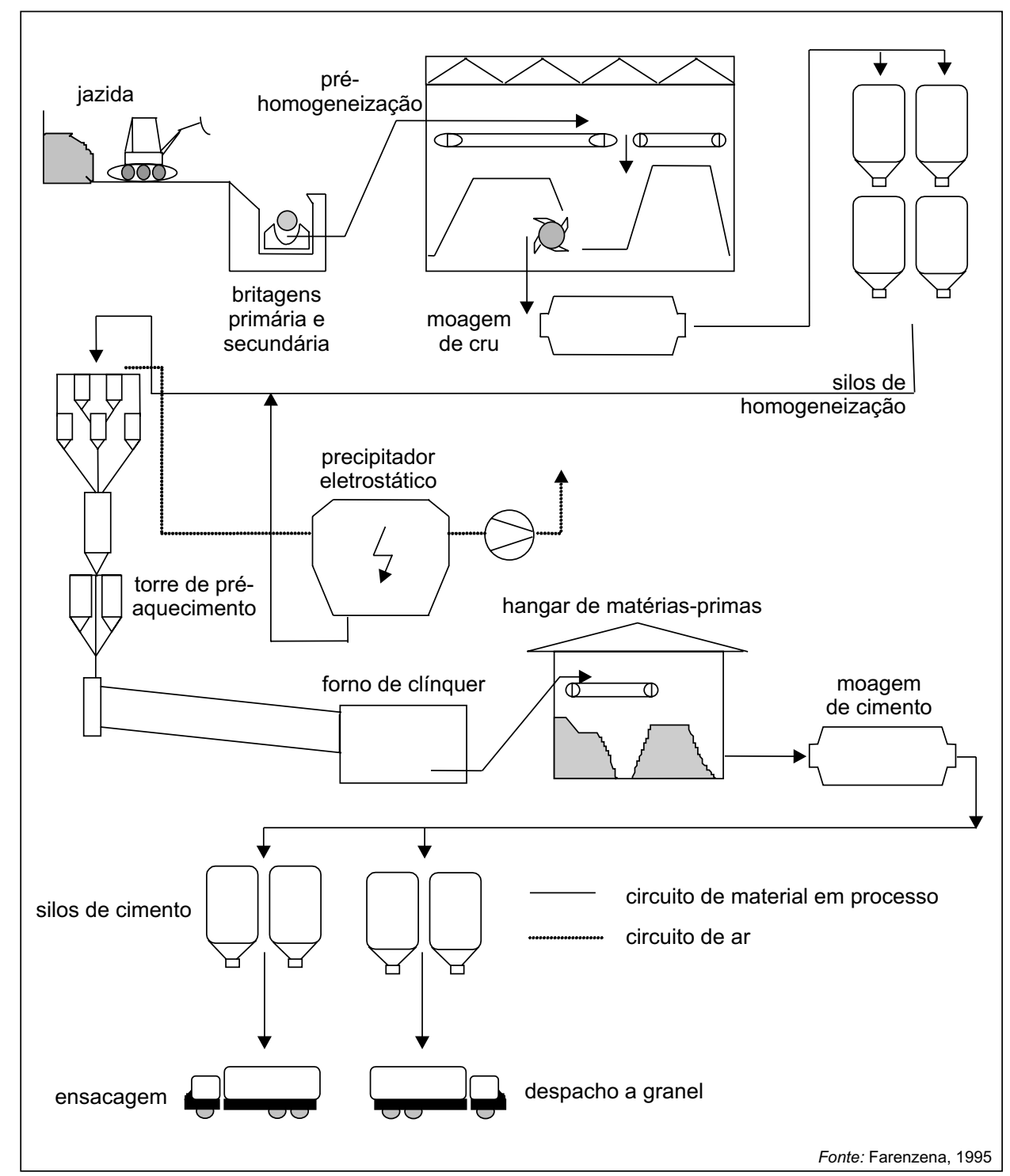

Figura 5 - Fluxograma de fabricação de cimento. 


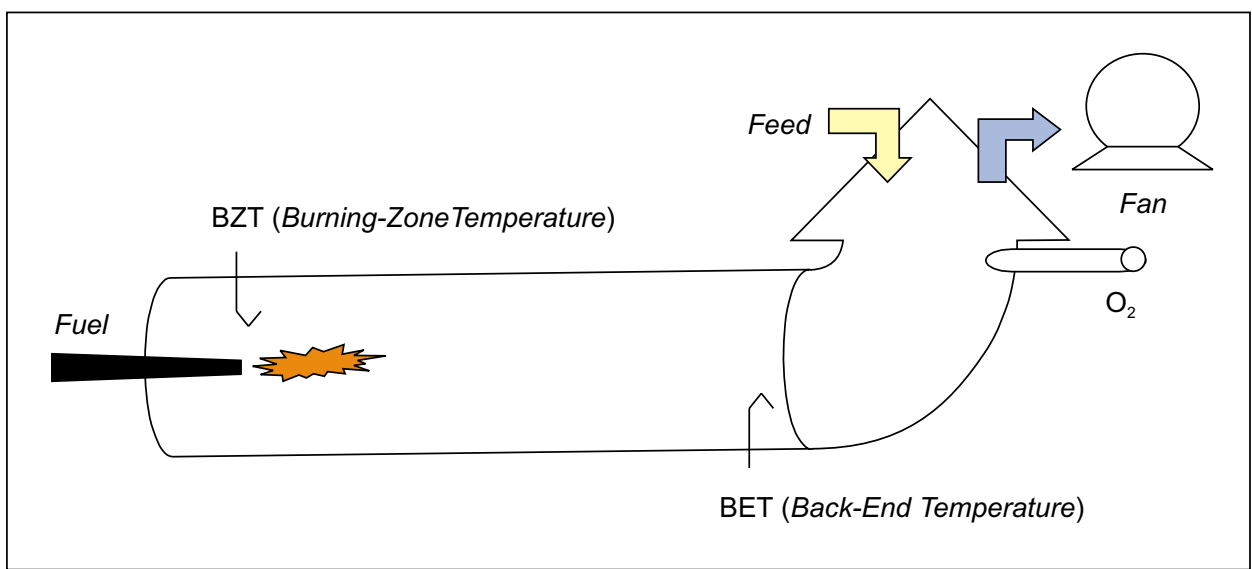

Figura 6 - Representação do processo de clinquerização e das variáveis intervenientes.

Os aspectos tecnológicos ligados ao hardware e ao software do controlador não são relevantes para o objetivo deste trabalho.

A BZT não é medida diretamente, sendo inferida por outras medições. Como o forno é rotativo, a corrente do motor elétrico de acionamento é proporcional ao torque resistente do conjunto. Devido à colagem interna, afetada pela temperatura, o torque resistente é proporcional à temperatura da zona de queima. Esta temperatura também é proporcional ao teor de $\mathrm{NO}_{x}\left(\mathrm{NO}+\mathrm{NO}_{2}\right)$, portanto pode ser inferida pela corrente do acionamento principal, pelo $\mathrm{NO}_{\mathrm{x}}$ ou por uma combinação de ambos. As demais grandezas podem ser medidas por instrumentação dedicada: analisadores de $\mathrm{O}_{2}$ e termo-elementos. A vazão de combustível e a alimentação de matéria-prima, em ton./hora, e a vazão de exaustão, em $\mathrm{Nm}^{3} /$ hora, são alteradas por atuadores dedicados, ligados ao controlador.

Em resumo, para o controlador de processo, se têm:

- entradas do controlador: BZT, $\mathrm{O}_{2}, \mathrm{BET}$;

- saídas do controlador: Fuel, Feed, Fan;

- variáveis auxiliares para o controle: $\Delta$ BZT, $\Delta \mathrm{O}_{2}, \Delta$ BET, $\Delta$ Fuel, $\Delta$ Feed, $\Delta$ Fan.

Pela abordagem de Mamdani, adaptada para o caso e ilustrada na Figura 7 (Tangerino, 1994), fuzzificam-se as variáveis de estado a partir das entradas. Para calcular os valores fuzzy se consideram as faixas nominais e os intervalos alocados ao universo de discurso de cada variável e as medições dos valores instantâneos e do gradiente das variáveis.

Obteve-se o bloco de regras de inferência e de defuzzificação por CBR. Lógicas similares operam em algumas dezenas de plantas no mundo. A lista dessas plantas é uma informação negocial relevante e deve ser omitida neste trabalho. As plantas possuem bases de regras, formadas pelo conhecimento de especialistas e práticas de produção. Ao equipar uma nova planta, o fabricante acessa as plantas atuais e, com base em parâmetros como a matéria-prima, o produto final, o tipo de forno e a capacidade de produção, escolhe plantas similares e resgata as bases, que serão adaptadas pelos especialistas para o caso atual.

Há diversas estratégias de controle: (i) regras válidas para operação instável do forno e situações de emergência; e (ii) regras válidas para operação estável. As regras para estabilidade são: (iia) bloco geral, que aumenta o feed até o valor desejado pelo especialista; (iib) bloco que mantém o feed e reduz o consumo de combustível, reduzindo o fuel; e (iic) bloco que opera ao atingir $100 \%$ do fan e que controla o feed e ofuel. 


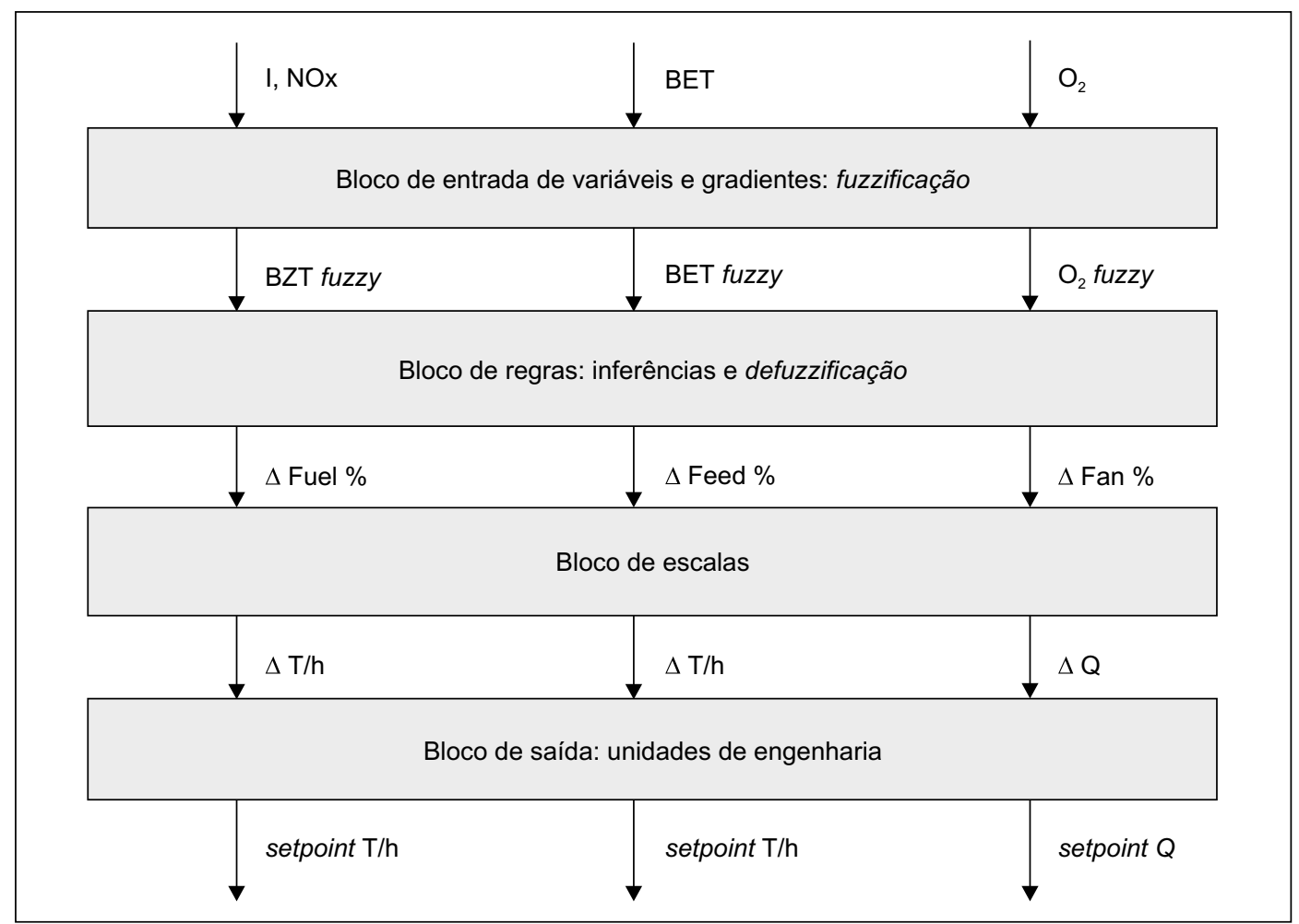

Figura 7 - Abordagem de Mamdani para a lógica de controle fuzzy. (Fonte: adaptado de Tangerino, 1994.)

O sistema parte segundo a estratégia (iia), associada à produtividade, até atingir o valor desejado de feed, quando assume a estratégia (iib), associada à redução de combustível. Se o fan atingir o valor máximo permitido, assume a estratégia (iic). O valor desejado de feed e o valor máximo de fan são informados pelo especialista.

As três variáveis de estado podem assumir três valores fuzzy cada, incorporando as tendências. Os especialistas definem como variar as variáveis de controle nos 27 casos possíveis de cada estratégia de controle. Por exemplo, para o caso geral, seja $\left(\mathrm{BZT}, \mathrm{O}_{2}, \mathrm{BET}\right)=(\mathrm{High}, \mathrm{OK}$, Low). Os especialistas devem responder: o que fazer com fuel, feed e fan se a temperatura da zona de queima está ficando alta, o oxigênio está ficando $\mathrm{OK}$ e a temperatura do fundo do forno está ficando baixa? Soluções iniciais são oferecidas pelos casos selecionados e os especialistas confirmam ou modificam uma solução. No caso exemplificado, os especialistas indicaram as seguintes ações: aumentar pouco o feed, reduzir pouco o fuel e reduzir muito o fan, representadas pelos valores $33,-33$ e $-67 \%$ (linha 6 da Tabela 1) de degraus das variáveis de controle, previamente especificados e revisados. Completa-se a tabela de modo similar, modificando-a conforme o resultado da estratégia ou a prática de produção. Após a revisão, o bloco de regras gerado entra para a base de casos, fechando o ciclo do $C B R$.

A seguir é exemplificada a lógica de controle com uma situação hipotética. Serão usadas funções de pertinência linear, combinações equiitativas entre a grandeza e seus gradientes e três níveis de fuzzificação para o entendimento da lógica. O controlador real emprega funções não-lineares e combinações diversas entre a grandeza e o gradiente. 
Tabela 1 - Bloco de regras geral para a clinquerização.

\begin{tabular}{|c|c|c|c|c|c|c|}
\hline \multicolumn{7}{|c|}{ Bloco de regras principais } \\
\hline & & & & \multicolumn{3}{|c|}{ Step $=$} \\
\hline & & & & $0,5 \mathrm{t} / \mathrm{h}$ & $0,05 \mathrm{t} / \mathrm{h}$ & $50 \mathrm{Nm}^{3} / \mathrm{h}$ \\
\hline caso & BZT $f$ & $\mathbf{O}_{2} f$ & BET $f$ & $\Delta$ Feed $\%$ & $\Delta$ Fuel $\%$ & $\Delta$ Fan $\%$ \\
\hline 1 & High & High & High & 100 & -67 & -67 \\
\hline 2 & & & OK & 67 & -67 & 0 \\
\hline 3 & & & Low & 33 & 0 & 100 \\
\hline 4 & & $\mathrm{OK}$ & High & 100 & 67 & 33 \\
\hline 5 & & & OK & 67 & -33 & 0 \\
\hline 6 & & & Low & 33 & -33 & -67 \\
\hline 7 & & Low & High & 100 & -100 & 0 \\
\hline 8 & & & OK & 67 & -67 & 67 \\
\hline 9 & & & Low & 33 & 0 & 100 \\
\hline 10 & OK & High & High & 67 & 0 & -67 \\
\hline 11 & & & OK & 33 & -33 & 0 \\
\hline 12 & & & Low & 0 & 67 & 33 \\
\hline 13 & & $\mathrm{OK}$ & High & 67 & 33 & 33 \\
\hline 14 & & & OK & 20 & 20 & 20 \\
\hline 15 & & & Low & 0 & 33 & 50 \\
\hline 16 & & Low & High & 0 & -50 & 0 \\
\hline 17 & & & OK & 0 & 0 & 50 \\
\hline 18 & & & Low & 0 & 33 & 67 \\
\hline 19 & Low & High & High & -33 & 33 & -100 \\
\hline 20 & & & OK & -67 & 100 & 0 \\
\hline 21 & & & Low & -100 & 100 & 0 \\
\hline 22 & & $\mathrm{OK}$ & High & -33 & 0 & -67 \\
\hline 23 & & & OK & -67 & 67 & 0 \\
\hline 24 & & & Low & -100 & 67 & 0 \\
\hline 25 & & Low & High & -33 & -100 & -33 \\
\hline 26 & & & OK & -67 & -33 & 0 \\
\hline 27 & & & Low & -100 & 33 & 33 \\
\hline
\end{tabular}

Sejam os valores nominais da Tabela 2 e a situação hipotética da Tabela 3, calculada pela função de pertinência linear da Figura 8, parametrizada pelos valores da Tabela 2 . Na coluna da direita da Tabela 3 se têm os valores já fuzzificados para as variáveis de entrada.
Agregando-se os primeiros e segundos termos das três variáveis de estado obtém-se o par:

- $\{(0,4 \mathrm{H} ; 0,6 \mathrm{OK} ; 0,5 \mathrm{H}) ;(0,6 \mathrm{OK} ; 0,4 \mathrm{~L}$; $0,5 \mathrm{OK})\}$. 
Tabela 2 - Faixas das variáveis necessárias ao controle no exemplo.

\begin{tabular}{ccc}
\hline Variável & Limites da variável & Faixa do gradiente \\
\hline $\mathrm{BZT}$ & $1.250^{\circ} \mathrm{C}$ a $1.450^{\circ} \mathrm{C}$ & $\Delta \mathrm{BZT} / \mathrm{min} .=-3 \% \mathrm{a}+3 \%$ \\
$\mathrm{O}_{2}$ & $0 \mathrm{a} 3 \%$ & $\Delta \mathrm{O}_{2} / \mathrm{min} .=-5 \% \mathrm{a}+5 \%$ \\
$\mathrm{BET}$ & $350^{\circ} \mathrm{C}$ a $450^{\circ} \mathrm{C}$ & $\Delta \mathrm{BET} / \mathrm{min} .=-3 \% \mathrm{a}+3 \%$ \\
Fuel & $0-5 \mathrm{t} / \mathrm{h}$ & \\
Feed & $0-100 \mathrm{t} / \mathrm{h}$ & Não se aplica \\
Fan & $0-5.000 \mathrm{Nm}^{3} / \mathrm{h}$ & \\
Todas & 3 níveis de fuzzificação $=>\mathrm{H}, \mathrm{OK}, \mathrm{L}$ (high, OK, low $)$ \\
\hline
\end{tabular}

Tabela 3 - Dados para o exemplo hipotético.

\begin{tabular}{cccccc}
\hline Variável & Instantânea & Fuzzy inst. & Gradiente & Fuzzy grad. & Fuzzy total \\
\hline Entrada: $\mathrm{BZT}$ & $1.350^{\circ} \mathrm{C}$ & $1 \mathrm{OK}$ & $2,4 \%$ & $0,8 \mathrm{H}+0,2 \mathrm{OK}$ & $0,4 \mathrm{H}+0,6 \mathrm{OK}$ \\
Entrada: $\mathrm{O}_{2}$ & $1,5 \%$ & $1 \mathrm{OK}$ & $-4 \%$ & $0,2 \mathrm{OK}+0,8 \mathrm{~L}$ & $0,6 \mathrm{OK}+0,4 \mathrm{~L}$ \\
Entrada: $\mathrm{BET}$ & $400^{\circ} \mathrm{C}$ & $1 \mathrm{OK}$ & $3 \%$ & $1 \mathrm{H}$ & $0,5 \mathrm{H}+0,5 \mathrm{OK}$ \\
Saída: Fuel & $3,5 \mathrm{t} / \mathrm{h}$ & & & & \\
Saída: Feed & $88 \mathrm{t} / \mathrm{h}$ & & & Não se aplica & \\
Saída: Fan & $4.500 \mathrm{Nm}^{3} / \mathrm{h}$ & & & & \\
\hline
\end{tabular}

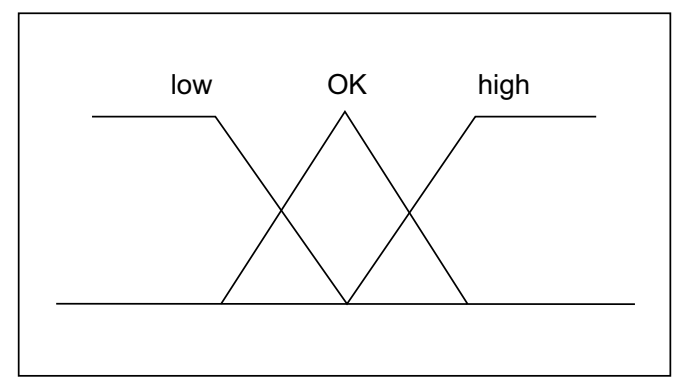

Figura 8 - Função de pertinência para as variáveis intervenientes no exemplo.

O primeiro elemento do par remete à linha 4 da Tabela 1 (BZT $\mathrm{H}, \mathrm{O}_{2}$ OK, BET H) e o segundo elemento remete à linha 17 da Tabela 1 (BZT OK, $\mathrm{O}_{2}$ L, BET OK). Dessas linhas (1 $1^{\mathrm{a}}$ parcela, 100, 67, 33, e 2 ${ }^{\mathrm{a}}$ parcela, $\left.0,0,50\right)$ se obtêm as contribuições para as variações impostas aos setpoints das variáveis de controle naquele instante. As contribuições são ponde- radas pela soma dos graus de pertinência de cada variável ao respectivo conjunto.

- $\Delta$ setpoint Feed $\%=(0,4+0,6+0,5) *$ $100+(0,6+0,4+0,5) * 0=150 \%$

- $\Delta$ setpoint Fuel $\%=(0,4+0,6+0,5) *$ $67+(0,6+0,4+0,5) * 0=100 \%$ 
- $\Delta$ setpoint Fan $\%=(0,4+0,6+0,5) *$ $33+(0,6+0,4+0,5) * 50=125 \%$

Por fim, tem-se a conversão das variáveis nas novas unidades de engenharia:

- $\Delta$ Feed $=150 \% * 0,5 \mathrm{t} / \mathrm{h}=0,75 \mathrm{t} / \mathrm{h}$, Feed inicial $=88 \mathrm{t} / \mathrm{h}$, novo Feed $=88,75 \mathrm{t} / \mathrm{h}$

- $\Delta$ Fuel $=-100 \% * 0,05 \mathrm{t} / \mathrm{h}=0,05 \mathrm{t} / \mathrm{h}$, Fuel inicial $=3,5 \mathrm{t} / \mathrm{h}$, novo Fuel $=3,55 \mathrm{t} / \mathrm{h}$

- $\Delta$ Fan $=125 \% * 50 \mathrm{Nm}^{3} / \mathrm{h}=62,5 \mathrm{Nm}^{3} / \mathrm{h}$, Fan inicial $=4.500 \mathrm{Nm}^{3} / \mathrm{h}$, novo $F a n=$ $4.562 \mathrm{Nm}^{3} / \mathrm{h}$.

A lógica de controle combina dois casos selecionados em um universo de discurso de 27 casos, formando a solução. O sistema também apresenta capacidade de aprendizagem, a partir de testes das condições de contorno do sistema. Para testar o limite da produtividade aumentase o valor desejado. Ao atingir esse valor, assume-se a estratégia de redução do fuel, mantendo as demais variáveis até que uma condição de contorno seja atingida ou que uma estratégia de instabilidade assuma, o que deve ser evitado. A monitoração dos resultados e a eventual modificação dos valores de estratégias constituem um processo de aprendizado.

\section{Discussão dos resultados da aplicação}

A seguir discutem-se alguns resultados de campo da aplicação dessa tecnologia.

Um operador humano age contínua, assíncrona e aleatoriamente, ao decidir sobre mudanças nos setpoints. Uma operação baseada em sistema especialista terá outro ritmo, pois a varredura de execução e a transferência das decisões da máquina para o processo não é instantânea. Podem-se usar ciclos de 1 minuto para atualização de dados de entrada e de saída e de 3 ou 5 minutos para transferência da saída para o campo. Um aspecto a considerar na determinação desses ciclos é a oscilação nas variáveis que alterações excessivas combinadas com as inércias naturais do processo podem causar.
A experiência de campo do autor mostra ganhos em produtividade $(\mathrm{t} / \mathrm{h})$ e energéticos (Kcal/t) de até $3 \%$ a $5 \%$ em relação a operadores especialistas. $\mathrm{O}$ detentor da tecnologia aponta diversas aplicações com ganhos de até $6 \%$ a $10 \%$ em produtividade e de até $3 \%$ em eficiência energética. Outras experiências internacionais também são relatadas.

Vaas \& Krogbeumker (1994) relatam uma aplicação de sistema especialista em uma moagem de cimento na Alemanha, em que se obteve um aumento de produtividade de 3,0-3,1\% e uma economia de energia de $2,9 \%$ após o comissionamento. Nussbaumer (1994) relata um caso no México em que um sistema especialista de otimização de processo de clinquerização trouxe aumentos de $1 \%$ e $3 \%$ na produção diária, redução de $2 \%$ e $4 \%$ no consumo energético e redução de $12 \%$ e $16 \%$ na variabilidade dos requisitos de qualidade do clínquer. As vantagens relatadas decorrem principalmente da melhor resposta obtida por um sistema automatizado às variabilidades do processo, em oposição às incertezas do comportamento de especialistas humanos, principalmente em situações de emergências, períodos longos e contínuos de operação e necessidade de alta produtividade. A capacidade de aprender e de assumir riscos também auxilia na construção deste resultado. Não foram abordados neste trabalho os aspectos psicológicos e comportamentais de operadores especialistas.

\section{Conclusão}

A partir do exposto e da experiência de campo do autor e das referências, conclui-se que os sistemas especialistas, o $C B R$ e a lógica fuzzy podem ser empregados em sistemas de controle multivariável de processos na indústria de processo contínuo, com resultados satisfatórios.

Portanto, sugere-se que os engenheiros de produção procurem conhecer as técnicas citadas, não se restringindo ao caso estudado, mas investigando outros processos de decisão nos quais haja ambigüidades, incertezas e variabilidades, e não haja um modelo exato e replicável. 


\section{Agradecimentos}

Deseja-se reconhecer e agradecer aos referees pelas sugestões apresentadas. Seu meticuloso trabalho de análise permitiu organizar e apresentar o material existente de modo mais compreensível e consistente, qualificando o resultado final.

\section{Referências Bibliográficas}

AAMODT, A.; PLAZA, E. Case-Based Reasoning: foundational issues, methodological variations, and system approaches. AI Communications, IOS Press, v. 7, n. 1, p. 39-59. Disponível em: http:// www.iiia.csic.es/People/enric/AICom_ ToC.html>. Acesso em: out. 2002.

ARARIBOIA, G. Inteligência Artificial. Rio de Janeiro: Ed. LTC, 1988.

CONAI: Tutorial Inteligência Artificial. In: CONGRESSO NACIONAL DE AUTOMAÇÃO INDUSTRIAL. Anais... São Paulo, 1994.

CORCHADO, J. et al. Hybrid instance-based system for predicting ocean temperature. International Journal of Computational Inteligence and Applications, v. 1, n. 1, p. 35-52, 2001.

FARENZENA, H. Fabricação de cimento portland. Porto Alegre: Ed. Cimentec, 1995.

KACPRZYK, J. Multistage fuzzy control. Chichester, UK: John Wiley Sons, 1997.

MCCARTHY, J. What is Artificial Intelligence? Disponível em: <http://www-formal.stanford.edu/ jmc/whatisai/whatisai.html>. Acesso em: out. 2002.
NIKOLOPOULOS, C. Expert systems: New York: Marcel Dekker, Inc., 1997.

NUSSBAUMER, A. LINKman II: Experience in the Apasco Group. In: 33 ${ }^{\text {th }}$ Technical Meeting/10 ${ }^{\text {th }}$ Aggregates and Ready-Mixed Concrete Conference. Basel, 1994.

OLIVEIRA JR., H. Lógica difusa: aspectos práticos e aplicações. Rio de Janeiro: Ed. Interciência, 1999.

RABUSKE, R. Inteligência Artificial. Florianópolis: Editora da UFSC, 1995.

TANGERINO, C. Lógica fuzzy aplicada em controladores programáveis. In: CONGRESSO NACIONAL DE AUTOMAÇÃO INDUSTRIAL, CONAI. Anais... São Paulo, 1994.

VAAS, M.; KROGBEUMKER, H. Das mühlenregelungssystem MCE bei Phoenix Zement in Beckum. ZKG Zement-Kalk-Gips International Forshung-Herstellung-Verwendung, n. 9, 1994, Bauverlag GmbH, Wiesbaden, 1993.

\section{ARTIFICIAL INTELLIGENCE: AN APPLICATION IN A CONTINUOUS PROCESS INDUSTRY}

\section{Abstract}

This paper describes an application of the fuzzy logic control and CBR in the continuous process industry. The techniques are discussed inside the larger branch of knowledge called Artificial Intelligence (AI), which can be related with the decision-making process in companies. Artificial Intelligence is pinpointed as a science that can support decisions in an easier and more precisely way than others methods as models and process indicators management. The steps for the erection of an Expert System, built mainly from human empirical experiences, are also discussed. The paper comes to an end by presenting a decision-making routine in a thermochemical process, in the cement industry, as performed by an Expert System decision-maker, based in CBR and fuzzy logic, and leading to a discussion about results and performance gains, in comparison with human-guided action in the same process.

Key words: fuzzy logic in continuous process industry, Artificial Intelligence in industrial engineering, expert systems in industrial engineering, $C B R$ in industrial engineering. 